6-1998

\title{
Scaled-Energy Floquet Spectroscopy in a Strong Electric Field: A Semiquantal Calculation of the Recurrence Spectrum
}

\author{
V. Kondratovich \\ William \& Mary \\ John B. Delos \\ William \& Mary, jbdelos@wm.edu
}

Follow this and additional works at: https://scholarworks.wm.edu/aspubs

Part of the Physics Commons

\begin{abstract}
Recommended Citation
Kondratovich, V. and Delos, John B., Scaled-Energy Floquet Spectroscopy in a Strong Electric Field: A Semiquantal Calculation of the Recurrence Spectrum (1998). Physical Review A, 57(6), 4604-4615. https://doi.org/10.1103/PhysRevA.57.4604
\end{abstract}

This Article is brought to you for free and open access by the Arts and Sciences at W\&M ScholarWorks. It has been accepted for inclusion in Arts \& Sciences Articles by an authorized administrator of W\&M ScholarWorks. For more information, please contact scholarworks@wm.edu. 


\title{
Scaled-energy Floquet spectroscopy in a strong electric field: A semiquantal calculation of the recurrence spectrum
}

\author{
Vladimir Kondratovich and John B. Delos \\ Department of Physics, College of William and Mary, Williamsburg, Virginia 23187
}

(Received 25 September 1997)

\begin{abstract}
We consider a hydrogen atom in a strong static electric field with a weak parallel radio-frequency (rf) field. We compute the photoabsorption spectrum by calculating the spectrum of Floquet states, including their quasienergies and their oscillator strengths. Our calculation is based upon "semiquantal" formulas: we calculate the discrete spectrum of quasienergy states by using a quantum adiabatic approximation combined with semiclassical (Bohr-Sommerfeld) quantization rules. We express this spectrum in a manner consistent with the method of scaled-variable spectroscopy, and then calculate the Fourier transform. These calculated absorption spectra and recurrence spectra are in good agreement with experiments on Li atoms. Additional approximations show that the recurrence spectrum is approximately equal to the product of the recurrence spectrum in a static field times an envelope function. That envelope function is the Fourier transform of a cluster of sidebands surrounding a progenitor level in the rf field. The resulting formula agrees with the low-frequency limit of a formula obtained from a semiclassical treatment. [S1050-2947(98)07406-X]
\end{abstract}

PACS number(s): 32.60. $+\mathrm{i}, 32.80 . \mathrm{Rm}$, 03.65.Sq

\section{INTRODUCTION}

In a recent experiment [1], the absorption spectrum of $\mathrm{Li}$ atoms was measured in the presence of a combined static and oscillating electric field. First the Li was excited $2 s \rightarrow 2 p$ $\rightarrow 3 s$, and then excited again by a linearly polarized tunable laser into high Rydberg states having a principal quantum number of approximately 100-130 and magnetic quantum number $m=0$ (polarization parallel to the static electric field). This absorption spectrum was measured by the scaledvariables method (varying the energy $E$ and the electric-field strength $F_{0}$ to keep the scaled energy $\epsilon=E / F_{0}^{1 / 2}$ fixed, and plotting absorption versus $w=F_{0}^{-1 / 4}$ ). Similar measurements (but not using scaled variables) have been made by Zhang et al. $[2,3]$.

The absolute square of the Fourier transform of the absorption spectrum is the "recurrence spectrum"; it has peaks corresponding to the classical actions of closed orbits of the electron, and the height of each peak is called the "recurrence strength" of that orbit. This recurrence spectrum was measured with a static field, and then with increasing strengths of the oscillating field, polarized parallel to the static field. With the static field only, the recurrence spectrum consists of a sequence of strong, nearly equally spaced peaks. As the strength of the oscillating field was increased, most of these recurrence peaks were reduced in a systematic fashion, but certain peaks remained: it was found that recurrence peaks would survive if the return time of the associated classical orbit was a multiple of the period of the oscillating field.

This phenomenon was given an explanation in [1] by extending "closed-orbit theory" [4] to time-dependent systems. We call closed-orbit theory a "semiclassical" theory; in that method we calculate recurrence spectra directly from three-dimensional classical trajectories. In the present paper we approach the phenomena from a complementary perspective, which we call a "semiquantal" framework. We sepa- rate variables in semiparabolic coordinates and use a WKB approximation to obtain quantized energy levels in the static field. We also use a semiclassical formula for the oscillator strength of each quantum state. We then consider the effect of the oscillating field on these levels and strengths in an adiabatic approximation. The oscillating field splits each energy level into clusters of sidebands, called "quasienergy states." Then the Fourier transform of this scaled absorption spectrum gives us finally the recurrence spectrum.

We call this approach "semiquantal" partly to distinguish it from the semiclassical ideas used in closed-orbit theory. In the present approach, although we use the WKB approximation, the focus is on quantized energy levels, and on their associated quasienergy states in the oscillating field. Thus we see more of quantum mechanics in the concepts and spirit of the present approach than appears in the implementation of closed-orbit theory.

The plan of the paper is as follows.

In Sec. II A, the WKB approximation is used to calculate the quantized energy levels in the static field [5] and a recently developed semiclassical formula [6] is used to calculate the associated oscillator strengths. These static-field levels are "progenitors" of quasienergy states in the oscillating field.

In Sec. II B, quasienergy states are calculated using a " diagonal" approximation, which is most appropriate if the frequency of the rf field is low compared to the frequencies of the unperturbed system (these approximations might also be valid more generally). The energy of each progenitor oscillates sinusoidally in time at the frequency of the rf field, with an amplitude proportional to the dipole moment of that quantum state. This time dependence constitutes a "frequency modulation" of the time dependence of the quantum state, which splits the progenitor into sidebands. The sidebands are equally spaced in energy, and they are weighted by Bessel functions. Thus each progenitor is split into a cluster.

The spectrum can be calculated in the standard way (fixed field strengths, varying energies), or in the scaled-variables 
framework. These calculations are in good agreement with the measurements (Sec. III).

Further interpretation requires additional approximations (Sec. IV). We show that the scaled-variables absorption spectrum has an enhanced periodicity compared to the unscaled spectrum (Sec. IV A), and we show that the sidebands are again nearly equally spaced in the scaled spectrum (Sec. IV B). If we make the approximation that all of the clusters have the same structure, then the absorption spectrum in the rf field becomes a convolution of the static spectrum with the spectrum of a single cluster (Sec. IV C). Therefore the Fourier transform of the absorption spectrum is a product of the static Fourier transform times the Fourier transform of a single cluster. Thus the static recurrence spectrum is multiplied by an envelope function.

That envelope function contains two parameters: one is related to the strength of the sidebands relative to the progenitor (and to the number of significant sidebands), and the other is related to the spacing of the sidebands. The net result is that those recurrence peaks are preserved that have return times close to a multiple of the period of the driving field. Finally, in Sec. IV D we show that the simplified semiquantal formula agrees with the low-frequency limit of semiclassical theory [1].

Some analytical expressions used in these semiquantal calculations are given in the Appendix.

\section{FLOQUET SPECTRUM IN STRONG DC AND WEAK rf FIELDS}

In the presence of an external homogeneous static electric field $F$ directed along the $z$ axis, the Hamiltonian of a hydrogen atom takes the form

$$
H_{\text {static }}=\frac{p^{2}}{2}-\frac{1}{r}+F_{0} z .
$$

Hereafter we use atomic units $\left(\hbar=1, e=1, m_{e}=1\right)$. If, in addition, an rf field polarized along the static one is present, the time-dependent Hamiltonian is

$$
H(t)=H_{\text {static }}+F_{1} z \cos \omega t
$$

with $F_{1}$ denoting the amplitude and $\omega$ the frequency of the $\mathrm{rf}$ field. In order to be as close as possible to the experiment [1] on the $\mathrm{Li}$ atom, we take the initial state to be the $3 s$ state of hydrogen, and we consider the case that the electric field of the laser is also polarized parallel to the static electric field, so that $m_{l}=L_{z} / \hbar=0$.

\section{A. Absorption spectrum in a static field}

Consider first the spectrum of the Hamiltonian (2.1). The potential energy has a saddle point at $z=-1 / \sqrt{F_{0}}, E_{s}=$ $-2 \sqrt{F_{0}}$. Below this saddle energy, the electric field $F_{0}$ splits the unperturbed Coulomb energy levels into manifolds of quasidiscrete levels. Above this energy $E=E_{s}$, the higherenergy quasidiscrete levels of each manifold survive, but the lower-energy levels in each manifold are broadened into a smooth continuum.

The quasidiscrete levels can be calculated from semiclassical quantization conditions [6]. This makes use of the sepa- rability of Hamiltonian (2.1) in semiparabolic coordinates

$$
u^{2}=r+z, \quad v^{2}=r-z
$$

Each state can be labeled by two parabolic quantum numbers $\left(n_{1}, n_{2}\right)$ (the magnetic quantum number $m$ equals zero). The Bohr-Sommerfeld quantization conditions take the form

$$
\begin{aligned}
& I_{u}(w, \epsilon, \beta)=\frac{1}{\pi} \int_{0}^{u_{0}} \sqrt{2(1+\beta)+2 E u^{2}-F_{0} u^{4}} d u=n_{1}+\frac{1}{2}, \\
& I_{v}(w, \epsilon, \beta)=\frac{1}{\pi} \int_{0}^{v_{0}} \sqrt{2(1-\beta)+2 E v^{2}+F_{0} v^{4}} d v=n_{2}+\frac{1}{2},
\end{aligned}
$$

where $\left(u_{0}, v_{0}\right)$ are the turning points of the $u$ or $v$ motions (first zero of the integrand); $\beta$ is the separation constant, $-1 \leqslant \beta \leqslant 1$. These equations can be rewritten in the scaled form:

$$
\begin{aligned}
& I_{u}(w, \epsilon, \beta)=w S_{u}(\epsilon, \beta)=n_{1}+\frac{1}{2}, \\
& I_{v}(w, \epsilon, \beta)=w S_{v}(\epsilon, \beta)=n_{2}+\frac{1}{2}
\end{aligned}
$$

with the scaled energy $\epsilon$ and scaled variable $w$ defined as

$$
\epsilon=E / \sqrt{F_{0}}, \quad w=F_{0}^{-1 / 4} .
$$

The scaled action variables $S_{u}$ and $S_{v}$ have convenient expressions through the elliptic integrals of Jacobi (see Appendix).

The two quantization conditions (2.4) and (2.5) lead to quantized values of both $\epsilon$ and $\beta$ (or $E$ and $\beta$ ). The separation constant $\beta$ has an important physical meaning. If we write $\beta_{\mathbf{n}}=\cos \theta_{\mathbf{n}}$, then $\theta_{\mathbf{n}}$ is the angle at which the $\mathbf{n t h}$ eigentrajectory intersects the nucleus; i.e., an electron ejected from the nucleus with energy $E_{\mathbf{n}}$ at angle $\theta_{\mathbf{n}}$ from the $z$ axis will find itself traveling on the $n$th eigentrajectory.

Figure 1 illustrates the development of a Stark manifold when the external electric field $F_{0}$ increases from zero. For small $F_{0}$, each level in the Stark manifold diverges linearly from its pure-Coulomb limit. As $F_{0}$ increases, we see significant curvature. The semiclassical system of Eqs. (2.6) and (2.7) defines the position of quasidiscrete levels for strong external fields, when the linear (first-order perturbation theory) approximation does not give reliable results. Each Stark level ends at a critical electric field strength where it reaches the top of an effective potential-energy barrier in the $v$ coordinate. For electric fields greater than the critical value (which depends on $n_{1}$ and $n_{2}$ ), each level broadens rapidly and disappears into the continuum.

Figure 1 also shows that well above the saddle energy $E_{s}$, and all the way up to $E=0$, there are many peaks belonging to the quasidiscrete spectrum. These peaks are a dominant structure of the absorption spectrum in this region, and they are the structure that produces the recurrences. Therefore, for this paper we can ignore the continuous background, and pay primary attention to these quasidiscrete lines in the absorption spectrum. 


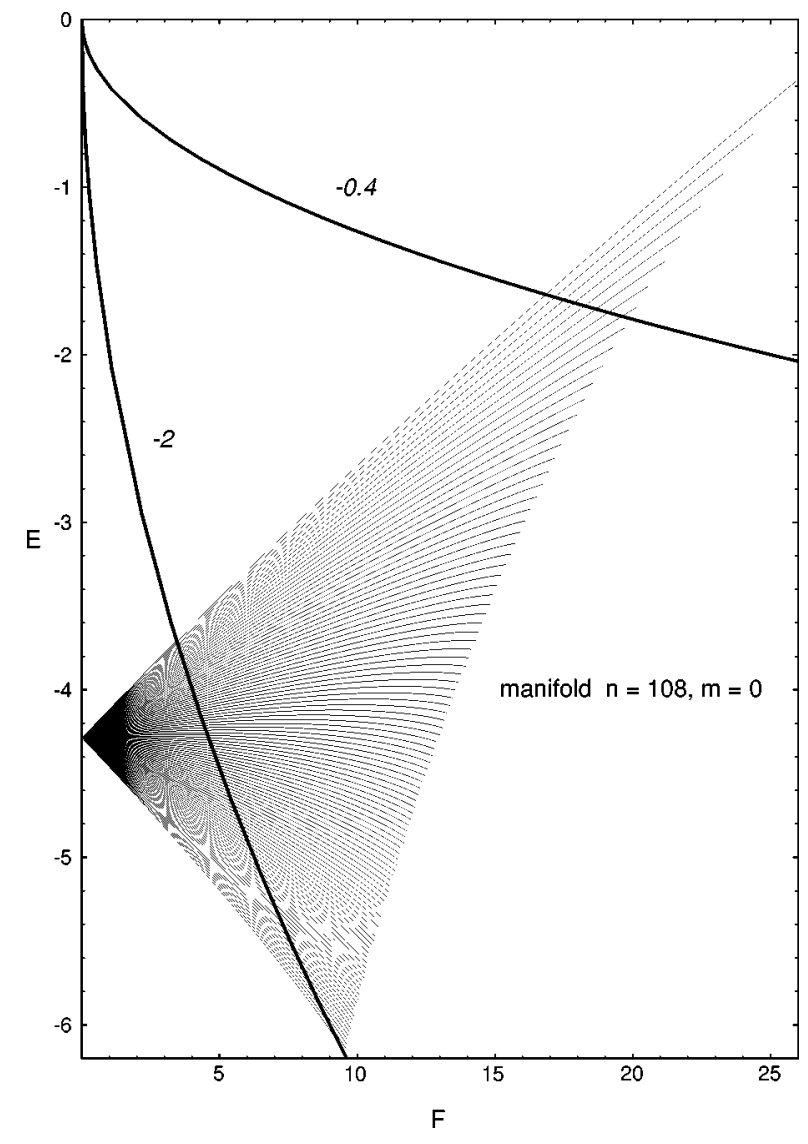

FIG. 1. Energies of a manifold of Stark states as a function of electric field strength (see Sec. II A). The levels are cut off when they start to rapidly broaden. Two lines of fixed scaled energy $\epsilon$ $=E / \sqrt{F}$ are shown. The energy of the saddle point corresponds to $\epsilon=-2$. Most of the present calculations and measurements were made near $\epsilon=-0.4$, where only a few "uphill" states survive. Energy $E$ is given in $10^{-5}$ a.u. and static field strength $F_{0}$ in $10^{-10}$ a.u.

The oscillator-strength density $D f$ for the transition from an initial state $\mathbf{i}$ to the Stark state $\mathbf{n}=\left(n_{1}, n_{2}\right)$ by $z$-polarized light is given by the formula

$$
D f_{\text {static }}(E)=\sum_{\mathbf{n}} f_{n_{1}, n_{2}}^{\mathbf{i}} \delta\left(E-E_{\mathbf{n}}\right)
$$

where the oscillator strength is defined as

$$
f_{n_{1}, n_{2}}^{\mathbf{i}}=2\left(E_{\mathbf{n}}-E_{\mathbf{i}}\right)\left|\left\langle\psi_{\mathbf{n}}|D| \psi_{\mathbf{i}}\right\rangle\right|^{2}
$$

with $D$ denoting here the dipole operator.

In another paper, we show that this oscillator strength can be written in a different form,

$$
f_{n_{1}, n_{2}}^{\mathbf{i}}=8 \pi\left(E_{\mathbf{n}}-E_{\mathbf{i}}\right)\left|\frac{\partial(\beta, E)}{\partial\left(I_{u}, I_{v}\right)}\right|\left|\mathcal{Y}\left(\theta_{\mathbf{n}}\right)\right|^{2},
$$

where $|\mathcal{Y}(\theta)|^{2}$ is the function defined in closed-orbit theory as the angular distribution of outgoing waves [4], Eq. (5.13b). This function is evaluated at the quantized ejection angle of the nth eigentrajectory.

\section{B. Absorption spectrum in the oscillating field}

Let us consider the case that the frequency of the oscillating field is low compared to the orbital frequencies of the electron. Then an adiabatic approximation makes sense. Let $\psi_{n_{1} n_{2} F_{0}}(\mathbf{q})$ be an eigenfunction of Eq. (2.1) with eigenvalue $E\left(n_{1}, n_{2}, F_{0}\right)$. The adiabatic approximation tells us that the function

$$
\psi_{a d}(\mathbf{q}, t)=\psi_{n_{1} n_{2} F(t)}(\mathbf{q}) \exp \left[-i \int^{t} E\left(n_{1}, n_{2}, F\left(t^{\prime}\right)\right) d t^{\prime}\right]
$$

is an approximate solution to the time-dependent Schrödinger equation with Hamiltonian (2.2) [here $F(t)=F_{0}$ $\left.+F_{1} \cos \omega t\right]$.

If the oscillating field is weak, we may expand the energy eigenvalue in powers of $F_{1}$ near the static field strength $F_{0}$ :

$$
\begin{aligned}
E\left(n_{1}, n_{2}, F\left(t^{\prime}\right)\right)= & E\left(n_{1}, n_{2}, F_{0}+F_{1} \cos \omega t^{\prime}\right) \\
\approx & E\left(n_{1}, n_{2}, F_{0}\right) \\
& +\frac{\partial E\left(n_{1}, n_{2}, F_{0}\right)}{\partial F} F_{1} \cos \omega t^{\prime} .
\end{aligned}
$$

The derivative

$$
\partial E\left(n_{1}, n_{2}, F\right) / \partial F \equiv \bar{z}\left(n_{1}, n_{2}, F\right)
$$

is the static polarizability of the $n_{1}, n_{2}$ level. One might be tempted to replace it by its value at $F=0$, but for the relatively strong fields considered here, this approximation is not accurate (it gives errors up to 40\%). The Bohr-Sommerfeld quantization conditions allow us to find an analytical expression for this static polarizability in a strong electric field:

$$
\bar{z} \equiv \bar{z}\left(n_{1}, n_{2}, F\right)=\frac{\partial E\left(n_{1}, n_{2}, F\right)}{\partial F}=-\frac{\partial\left(I_{u}, I_{v}\right)}{\partial(F, \beta)} / \frac{\partial\left(I_{u}, I_{v}\right)}{\partial(E, \beta)} .
$$

All derivatives entering Eq. (2.15) have convenient expressions as Jacobi elliptic integrals, which allow easy and rapid calculation. These expressions can be found in the Appendix.

Substituting Eq. (2.13) into Eq. (2.12), we obtain a sin function in an exponent, which can be expanded in a Fourier series [3],

$$
\exp \left(-i\left(F_{1} \bar{z} / \omega\right) \sin \omega t\right)=\sum_{l=-\infty}^{+\infty} J_{l}\left(F_{1} \bar{z} / \omega\right) \exp (-i l \omega t)
$$

where, as usual, $J_{l}(x)$ denotes the Bessel function.

Now we make an additional approximation. We propose that the adiabatically adjusting function $\psi_{n_{1} n_{2} F(t)}(\mathbf{q})$ can be replaced by the eigenfunction in the static field $\psi_{n_{1} n_{2} F_{0}}(\mathbf{q})$. We can justify this because we will only use the eigenfunctions to calculate dipole overlap integrals $\left\langle n_{1} n_{2} F(t)|z| i\right\rangle$ in Eq. (2.10). These overlap integrals depend upon the form of the eigenfunction in a small region $\left(\sim 10 a_{0}\right)$ around the nucleus. The adiabatic eigenfunctions might change substan- 
tially near their outer turning points, but they do not change very much in this small region near the nucleus.

It follows that each adiabatic eigenfunction, together with its phase, can be interpreted as a superposition of quasienergy states,

$$
\begin{aligned}
\psi_{a d}(\mathbf{q}, t)= & \psi_{n_{1} n_{2} F_{0}}(\mathbf{q}) \sum_{l=-\infty}^{+\infty} J_{l}\left(F_{1} \bar{z} / \omega\right) \\
& \times \exp \left\{-i\left[E\left(n_{1}, n_{2}, F_{0}\right)+l \omega\right] t\right\}
\end{aligned}
$$

The superposition consists of a "progenitor'" state (the one with $l=0$ ) and a set of sidebands $l \neq 0$, equally spaced in quasienergy. In our approximation, the progenitor and the sidebands all have the same spatial dependence. The sidebands are weighted compared to the progenitor level by the Bessel functions,

$$
J_{l}^{2}(q): J_{0}^{2}(q)
$$

This ratio is determined by the parameter $q=F_{1} \bar{z} / \hbar \omega$, in other words, by the ratio of the maximal energy of a static dipole with moment $\bar{z}$ in the static field of strength $F_{1}$ to the energy interval $\hbar \omega$ between sidebands. For weak rf fields the amplitudes of sidebands are low, having the order of magnitude $q^{|l|} / 2^{|l|}|l|$ ! whereas for higher rf field strengths the progenitor level may even disappear. However, all intensities satisfy the sum rule [7]

$$
\sum_{l=-\infty}^{+\infty} J_{l}^{2}(q)=1
$$

for all values of $q$. That means that intensity (in particular, the absorption rate or oscillator strength) moves from the progenitor to the sidebands as $q$ increases, but the total intensity is conserved as the parameters of the rf field vary.

It follows that the absorption spectrum is split from the progenitor level into the sidebands. In the rf field the atom in the $3 s$ state will absorb a laser photon of frequency $\hbar \omega_{L}$ $=\mathcal{E}\left(n_{1}, n_{2}, l ; F_{0}\right)-E_{3 s}$ to go into a state of quasienergy

$$
\mathcal{E}\left(n_{1}, n_{2}, l ; F_{0}\right)=E\left(n_{1}, n_{2}, F_{0}\right)+l \omega
$$

The oscillator strength for this transition is the oscillator strength for the progenitor weighted by the Bessel functions,

$$
f_{n_{1}, n_{2}, l}^{\mathbf{i}}=f_{n_{1}, n_{2}}^{\mathbf{i}} J_{l}^{2}\left(F_{1} \bar{z} / \omega\right)
$$

where $f_{n_{1}, n_{2}}^{\mathbf{i}}$ is the oscillator strength for absorption into the $\left(n_{1}, n_{2}\right)$ state in the absence of the rf field.

According to the expressions (2.21), (2.20), (2.10), and (2.11) we can introduce the oscillator-strength density of the $l$ th sideband $(l=0$ here gives the strength for the band of $\mathrm{dc}$ progenitor levels) in the external rf field as

$$
\begin{aligned}
D f_{l}(E ; F)= & 8 \pi \sum_{n_{1}, n_{2}}\left(E-E_{\mathbf{i}}\right) J_{l}^{2}\left(\frac{F_{1} \bar{z}}{\omega}\right) \\
& \times\left|\frac{\partial(E, \beta)}{\partial\left(I_{u}, I_{v}\right)}\right|\left|\mathcal{Y}\left(\theta_{n_{1}, n_{2}, l}\right)\right|^{2} \\
& \times \delta\left\{E-\left[E\left(n_{1}, n_{2}, F_{0}\right)+l \omega\right]\right\} .
\end{aligned}
$$

The sum over the progenitor and sidebands gives us the oscillator-strength density in the presence of the rf field,

$$
D f_{\mathrm{rf}}(E)=\sum_{l=-\infty}^{+\infty} D f_{l}(E) \text {. }
$$

The final formula (2.23) gives a very simple picture of the quasidiscrete absorption spectrum in presence of the combined dc and rf fields. We just have to superimpose the spectral scan for the static field with equivalent scans that have been shifted along the energy axis by a multiple of $\hbar \omega$ and then properly weight the peaks. The weights primarily depend on $l$, the label of the sideband, but they have a slight dependence on $n_{1}, n_{2}$ and $F$ through Eq. (2.15) and through the dependence of $\theta_{n_{1}, n_{2}, l}$ on $n_{1}, n_{2}$.

The procedure for calculating the scaled-variable absorption spectrum is, then, the following. (i) Fixing $\epsilon$, find $w_{\mathbf{n}}$ and $\beta_{\mathbf{n}}=\cos \theta_{\mathbf{n}}$ satisfying the quantization conditions (2.6) and (2.7); these are the progenitor levels. (ii) For each, evaluate $\bar{z}\left(n_{1}, n_{2}, F_{0}\right)$ using Eq. (2.15) and the Appendix. Also evaluate $\left|\partial(E, \beta) / \partial\left(I_{u}, I_{v}\right)\right|$ (which is slowly varying) and $\mathcal{Y}\left(\theta_{\mathbf{n}}\right)$. This data in Eq. (2.22) gives the static spectrum. (iii) For $l \neq 0$, the quantization conditions are replaced by

$$
\begin{gathered}
\mathbf{I}\left(w_{\mathbf{n}, l}, \epsilon^{\prime}\left(\boldsymbol{\epsilon}, w_{\mathbf{n}, l}\right), \beta_{\mathbf{n}, l}\right)=\mathbf{n}+1 / 2, \\
\epsilon^{\prime}\left(\boldsymbol{\epsilon}, w_{\mathbf{n}, l}\right)=\epsilon-l \tilde{\omega} / w_{\mathbf{n}, l} .
\end{gathered}
$$

The above equations mean that we find the progenitor of the $l$ th sideband at a scaled energy $\epsilon^{\prime}$ that differs from the value $\epsilon$ fixed in the scaled spectrum (see Fig. 2).

In Eq. (2.22) we artificially broaden the $\delta$ functions to model the experimental resolution. Let

$$
\begin{gathered}
D f_{\text {static }}(w ; \epsilon)=D f_{\text {static }}(E(w ; \epsilon) ; F(w)), \\
D f_{l}(w ; \epsilon)=D f_{l}(E(w ; \epsilon) ; F(w)) \\
D f_{\mathrm{rf}}(w ; \epsilon)=\sum_{l=-\infty}^{+\infty} D f_{l}(w ; \epsilon)
\end{gathered}
$$

represent the scaled absorption spectrum obtained in this way. The recurrence spectrum is then the Fourier transform of $D f_{\mathrm{rf}}(w ; \epsilon)$ over a selected range of $w$,

$$
\begin{gathered}
R_{l}(s)=\int e^{-2 \pi i s w} D f_{l}(w ; \epsilon) d w \\
R_{\text {static }}(s)=\int e^{-2 \pi i s w} D f_{\text {static }}(w ; \epsilon) d w,
\end{gathered}
$$




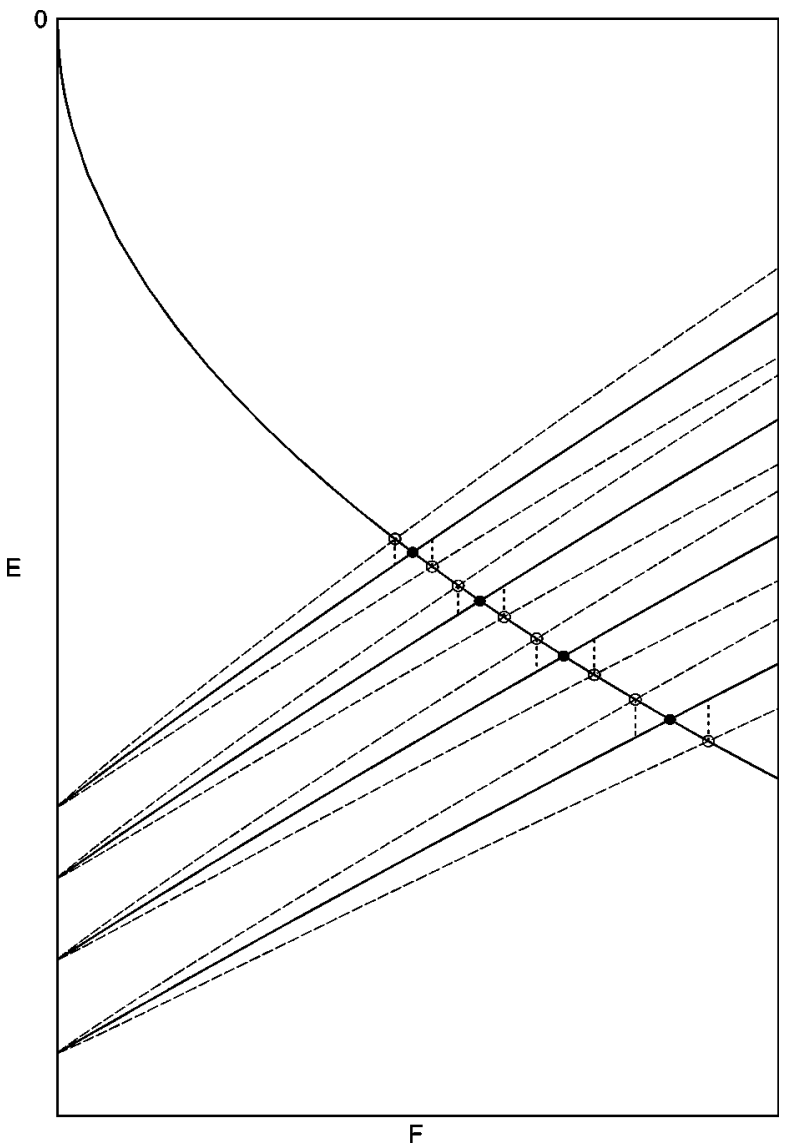

FIG. 2. Diagram representing the calculation of a scaled spectrum with sidebands (see Sec. II B). The heavy curve represents a fixed scaled energy. Four Stark levels are shown (solid lines), each with two sidebands (dashed lines). Each progenitor intersects the scaled-energy curve at a point marked by a solid dot. The corresponding value of $w=F^{-1 / 4}$ gives the location of this progenitor in the scaled spectrum. At any value of $F_{0}$, the (quasi)energy of the $l$ th sideband is related with the energy of its progenitor as $\mathcal{E}_{l}=E$ $+l \omega$. Because of the scaling method used, this spacing between progenitor and sidebands increases with $F$ as $F^{3 / 4}$. Each sideband intersects the scaled-energy curve at a point marked by a hollow dot. This connection is shown as vertical dashed lines. In scaled form, it adds another quantization condition [Eq. (2.25)] to the Bohr-Sommerfeld equations (2.24).

$$
R_{\mathrm{rf}}(s)=\int e^{-2 \pi i s w} D f_{\mathrm{rf}}(w ; \epsilon) d w=\sum_{l=-\infty}^{+\infty} R_{l}(s)
$$

(Sometimes it is convenient to put weighting functions or window functions into the Fourier transform, but in the present case we have not done this.)

Computed and measured recurrence spectra are in excellent agreement (see the next section).

\section{COMPARISON OF SEMIQUANTAL CALCULATIONS WITH EXPERIMENT}

The comparison of typical static $\left(F_{1}=0\right)$ scaled absorption spectra is presented in Fig. 3. We see generally good agreement, especially in the location of the peaks. The heights of the peaks in theory and experiment do not always
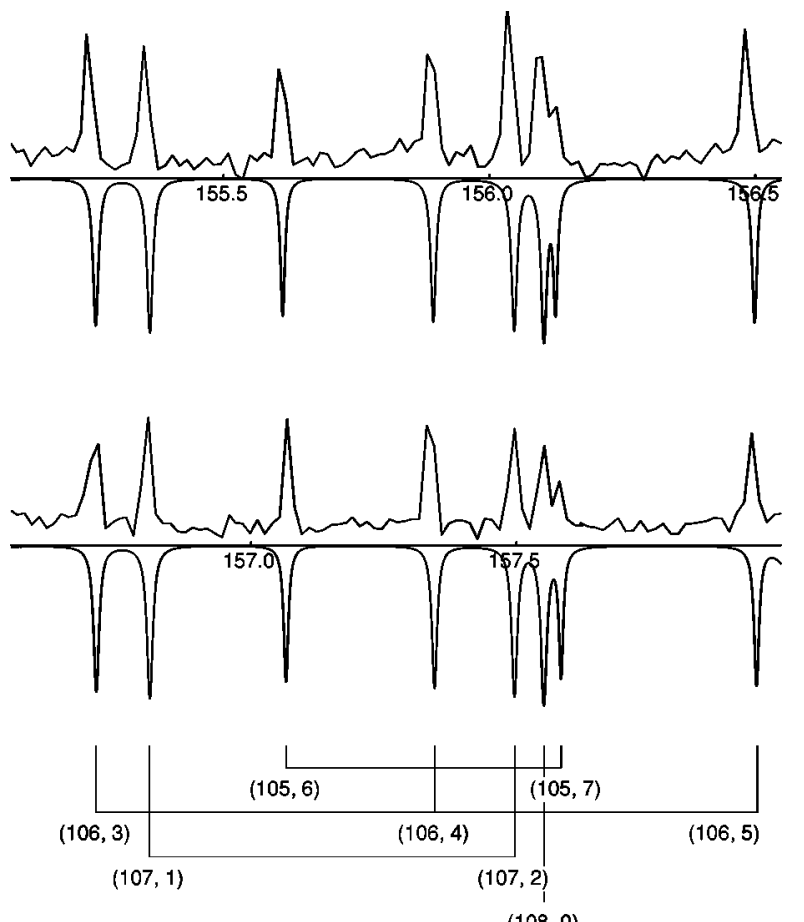

FIG. 3. The absorption spectrum is nearly periodic in the scaled variable $w=F_{0}^{-1 / 4}$; the period is the spacing between the $\left(n_{1}, 0\right)$ and the $\left(n_{1}+1,0\right)$ states. Here is the static absorption spectrum (no rf field) with two adjacent periods compared. Atomic units used. (The upper line on each graph represents the experimental data [8], lower is our calculation, with peaks artificially broadened to imitate the experimental resolution.) Quantum numbers are assigned in the lower graph.

agree so well, but this experiment was not optimized for the measurement of peak heights.

Both theoretical and experimental spectra are remarkably periodic in the scaled variable $w$. In order to show this, we superimpose in Fig. 3 two adjacent periods of theoretical and experimental spectra. This periodicity is explained in Sec. IV A.

The scaled absorption spectra in the presence of the rf field are also in good agreement, as shown in Fig. 4. However, we had to shift the experimental spectrum to the right by $\delta w=0.1$ to make it match the theory [9].

The experiment was designed for the measurement of recurrence spectra, and we find excellent agreement between theory and observation (Fig. 5). As the rf field strength $F_{1}$ increases, most of the recurrences are systematically weakened; our calculations show this effect quantitatively. Other comparisons between the present calculations, semiclassical calculations, and measurements were given in Figs. 3 and 4 of Ref. [1].

In Fig. 5, the recurrences that survive as $F_{1}$ increases are those for which the return time is a multiple of the rf period. This observation was given a semiclassical interpretation in Ref. [1]. The same observation can also be understood in the semiquantal perspective of the present paper. This interpretation is developed in the next section.

\section{INTERPRETATION OF RESULTS}

The semiquantal interpretation is based on the following facts, which we discuss in the following subsections: (i) The 

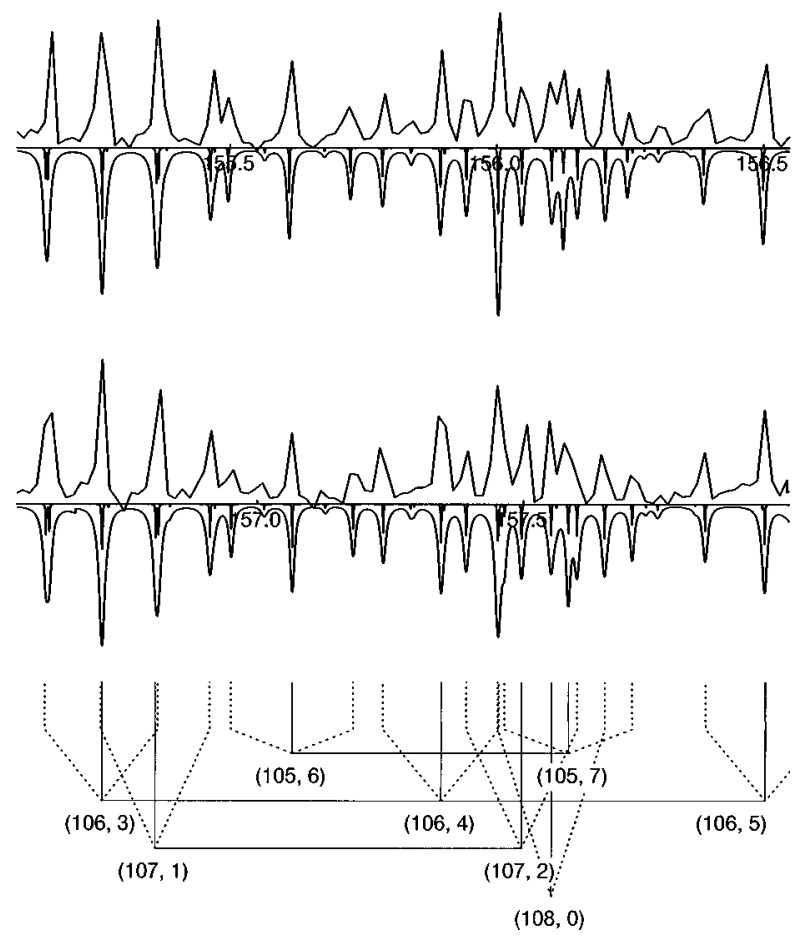

FIG. 4. The absorption spectrum (scaled energy $\epsilon=-0.4$ ) in the presence of the rf field with $\widetilde{F}_{1}=0.005$ and $\tilde{\omega}=1 / \pi \approx 0.32$. Two adjacent periods are shown. The experimental data [8] (the upper graphs) have been shifted by $\delta w=0.1$ (see Sec. III and Ref. [9]). Each progenitor has an easily visible pair of sidebands. Again quantum numbers are assigned to progenitors and first sidebands $(l=$ \pm 1 ) in the lower graph.

scaled absorption spectrum is characterized by enhanced periodicity in comparison with the unscaled spectrum; (ii) the scaled rf field generates sidebands that are nearly equally shifted relative to each other in the scaled spectrum; (iii) it follows that the scaled absorption spectrum is given by a formula that resembles a convolution sum; (iv) therefore, the recurrence spectrum is a product of the static recurrence spectrum times an envelope function.

\section{A. Periodicity of scaled spectra}

In order to illustrate the enhancement of periodicity in the scaled spectrum, let us consider the first-order formula for the energy levels in a static field [10],

$$
E\left(n_{1}, n_{2}, F_{0}\right)=-\frac{1}{2 n^{2}}+\frac{3}{2} n\left(n_{1}-n_{2}\right) F_{0},
$$

where $n=n_{1}+n_{2}+|m|+1$ is the principal quantum number. The Stark spectrum has an approximate multiperiodicity: for each fixed $n$, the levels are equally spaced, and the spacing between successive $n$ levels is nearly constant at large $n_{1}$. These energy gaps correspond to the two frequencies of motion of the electron, and Fourier transformation of the absorption spectrum as a function of energy would give peaks at the corresponding periods. However, both of these periods depend upon the energy, so the periodicities are not exact. We see this most clearly if we fix $n_{2}$ and vary $n_{1}$, for then both terms in Eq. (4.1) are quadratic in $n_{1}$.

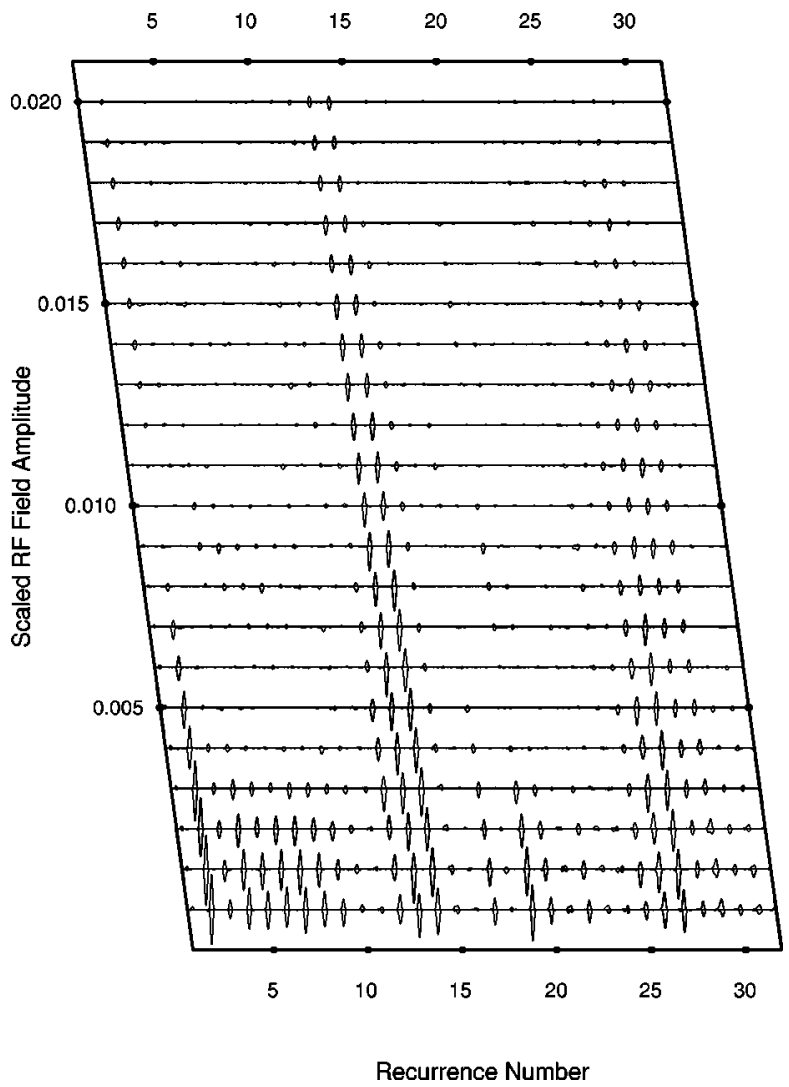

FIG. 5. Recurrence spectra (absolute squares of Fourier transforms of absorption spectra). They are drawn for various values of the scaled rf field amplitude $\widetilde{F}_{1}=F_{1} / F_{0}$ (mirror plot: theoretical peaks are plotted upright and experimental [8] are upside down.) The scaled rf frequency $\tilde{\omega}=0.32$ is in proportion $1: 14.5$ to the frequency of the parallel orbit. The graph shows the systematic weakening of most recurrences, but survival of the 14th and 28th peaks of the static recurrence spectrum. These repetitions of the parallel orbit allow the rf field to make an integer number of oscillations. This preservation rule becomes more selective when the rf field strength increases.

This multiperiodicity becomes more precise if the scaledvariable absorption spectrum is examined, and in a certain limit this spectrum becomes almost exactly periodic. In scaled variables, Eq. (4.1) looks as follows:

$$
\epsilon=-\frac{w^{2}}{2 n^{2}}+\frac{3}{2} \frac{n\left(n_{1}-n_{2}\right)}{w^{2}}
$$

with scaled parameters $\epsilon$ and $w$ defined in Eq. (2.8).

The scaled spectrum is the set of allowed, quantized values of $w_{n_{1} n_{2}}$ at any specified $\epsilon$. Consider the set of states having $n_{2}=0, n_{1}=n-1 \approx n$. For those states (the highestenergy, or uphill Stark states), Eq. (4.2) becomes

$$
\epsilon=-\frac{w^{2}}{2 n^{2}}+\frac{3}{2} \frac{n^{2}}{w^{2}} .
$$

We can solve this equation for $w_{n}(\epsilon)$, and we find $w_{n}(\epsilon)$ $=c(\epsilon) n$. The uphill states are evenly spaced in the scaled spectrum.

We already mentioned that this first-order formula (4.1) is insufficiently accurate for the field strengths considered here. 
It simply illustrates the enhancement of periodicity in the scaled-variables spectrum, and this enhanced periodicity holds also in more precise formulas. Specifically, in the semiclassical quantization conditions (2.6)-(2.7), let us set $n_{2}=0$, and then make the approximation $\beta \simeq 1$. We find again an evenly spaced set of eigenvalues,

$$
\begin{aligned}
w_{n_{1}} & =\left(n_{1}+1 / 2\right) / S_{u}(\epsilon, 1) \\
& \equiv\left(n_{1}+1 / 2\right) / S_{\|}(\epsilon),
\end{aligned}
$$

where $S_{\|}(\epsilon)=S_{u}(\epsilon, 1)$ is the scaled classical action of the uphill periodic orbit.

The above periodicity (which would hold exactly if $n_{2}$ were equal to $-1 / 2$ ) holds also in good approximation for small positive $n_{2} . \Delta w=1 / S_{\|}(\epsilon)$ is the largest "wavelength" in the scaled spectrum. Therefore the Fourier transform has a "fundamental" at $s=S_{\|}(\epsilon)$, and "overtones" of this fundamental. (In the present case the fundamental happens to be very weak.)

In addition, the eigenvalues are approximately evenly spaced in $n_{2}$. Equation (2.8) implies

$$
\epsilon=E\left(n_{1}, n_{2}, F_{0}\right) F_{0}^{-1 / 2}=w_{n_{1} n_{2}}^{2} E\left(n_{1}, n_{2}, w_{n_{1} n_{2}}^{-4}\right),
$$

and if we differentiate the right-hand side of this equation with respect to $n_{2}$ or $n_{1}$ holding $\epsilon$ fixed, we find

$$
2 w \frac{\partial w}{\partial n_{i}}+\frac{\partial E}{\partial F} \frac{\partial F}{\partial w} \frac{\partial w}{\partial n_{i}}=-\frac{\partial E}{\partial n_{i}} w^{2},
$$

So

$$
\begin{aligned}
\frac{\partial w / \partial n_{2}}{\partial w / \partial n_{1}} & =\frac{\partial E\left(n_{1}, n_{2}, F\right) / \partial n_{2}}{\partial E\left(n_{1}, n_{2}, F\right) / \partial n_{1}}=\frac{\partial H\left(I_{1}, I_{2}\right) / \partial I_{2}}{\partial H\left(I_{1}, I_{2}\right) / \partial I_{1}}=\frac{\omega_{2}\left(I_{1}, I_{2}\right)}{\omega_{1}\left(I_{1}, I_{2}\right)} \\
& \simeq \frac{\omega_{2}\left(I_{1}, 0\right)}{\omega_{1}\left(I_{1}, 0\right)} \equiv \frac{\omega_{2}\left(I_{1}, 0\right)}{\omega_{\|}} .
\end{aligned}
$$

The last approximation holds if $I_{2}$ is small. The scaled spectrum is defined in just such a way that the frequency ratio (4.9) is fixed, independent of $I_{1}$ at fixed $\epsilon$. Therefore the eigenvalues also have nearly constant spacing in $n_{2}$ [11].

In the case considered, $\epsilon=-0.4$, the ratio (4.9) is 0.5 . It follows that the sequence of states $\left(n_{1}, n_{2}=1\right)$ lies about halfway between the states $\left(n_{1}, n_{2}=0\right)$. These two sequences together give a Fourier peak at $s=2 S_{\|}$, which is one of the strongest peaks in the recurrence spectrum. (In semiclassical theory, we say that this happens because we are near a $2 / 1$ bifurcation of the parallel orbit.)

\section{B. Sideband spacing in scaled spectra}

The above periodicity is preserved when the rf field is applied, and another important periodicity appears.

The quasienergies of sidebands $\mathcal{E}\left(n_{1}, n_{2}, l ; F_{0}\right)$ are related with the energies of progenitors $E\left(n_{1}, n_{2}, F_{0}\right)$ by Eq. (2.20). In the scaled-variables measurement [1], the laser frequency, the static electric field strength $F$, the rf frequency $\omega$ and the rf field amplitude are changed simultaneously in order to keep fixed the scaled energy $\epsilon$ of the final state, the scaled rf frequency $\tilde{\omega}$ and the scaled rf field strength $\widetilde{F}_{1}$ :

$$
\begin{gathered}
\epsilon=\mathcal{E}\left(n_{1}, n_{2}, l ; F_{0}\right) F_{0}^{-1 / 2}=F_{0}^{-1 / 2}\left[E\left(n_{1}, n_{2}, F_{0}\right)+l \omega\right], \\
\tilde{\omega}=\omega F_{0}^{-3 / 4}, \quad \widetilde{F}_{1}=F_{1} / F_{0} .
\end{gathered}
$$

As the spectral peaks are always recorded at a fixed value of the scaled energy $\epsilon$, Eq. (2.20) can be rewritten, after division by $\sqrt{F_{0}}$, as

$$
\epsilon=w^{2} E\left(n_{1}, n_{2}, w\right)+l \tilde{\omega} / w .
$$

The values of scaled variable $w$ that satisfy Eq. (4.12) define the positions of the absorption peaks $w_{n_{1}, n_{2}, l}$. The derivative of $w_{n_{1}, n_{2}, l}$ with respect to $l$ gives the spacing between the peaks belonging to neighboring sidebands having the same quantum numbers $n_{1}$ and $n_{2}$. By the same kind of reasoning that was used to obtain Eq. (4.9), we obtain

$$
\frac{\partial w / \partial l}{\partial w / \partial n_{1}}=\frac{\tilde{\omega} / w^{3}}{\partial E / \partial n_{1}}=\frac{\omega}{\omega_{1}\left(I_{1}, I_{2}\right)} \simeq \frac{\omega}{\omega_{\|}},
$$

which is again constant in the scaled spectrum. Therefore the rf field brings a third constant period into the scaled spectrum. The spacing of sidebands is to the fundamental spacing as the rf frequency is to the frequency of the parallel orbit. (There is a subtlety here: in Eq. (4.13) $\omega_{\|}$is the frequency of the parallel orbit not at the selected value of $\epsilon$, but at $\epsilon^{\prime}$ $=\epsilon-l \tilde{\omega} / w$. In the present case, this difference is negligible.)

Combining Eq. (4.13) with the expression (4.5) for the spacing of progenitors, we find that the sideband spacing, which is $\omega$ in the unscaled spectrum, is in the scaled spectrum given by

$$
a=\frac{\partial w}{\partial l}=\frac{\tilde{\omega}}{\tilde{\omega}_{\|} S_{\|}}=\frac{T_{\|}}{T_{\mathrm{rf}} S_{\|}} \equiv \frac{1}{s_{s b}} .
$$

The formula (4.14) represents the main result of this subsection. As the scaled action for the parallel orbit $S_{\|}$is constant in the scaled spectrum, this formula tells us that all neighbor sidebands are equally displaced along the $w$ axis with the distance between them equal to $a$. This new periodicity must show up in the Fourier spectrum at actions $s_{s b}$ $=S_{\|} T_{\mathrm{rf}} / T_{\|}$. What will it do to the recurrence spectrum?

\section{Recurrence spectrum}

In the previous section we have shown that the $l$ th sideband is displaced from the progenitor band by a constant shift equal to $l a$ along the $w$ axis. Now we discuss the effect of these evenly shifted sidebands on the Fourier transform of the scaled spectrum. We will make several approximations that will introduce errors up to few percent in the result but will also allow us to emphasize the qualitative features of the effect.

Since we deal with states that are stretched along the external field (quantum numbers $n_{2} \ll n_{1}$ ), let us substitute for the static polarizability $\bar{z}$ Eq. (2.15) the value $\bar{z}_{\|}$for the parallel orbit. We will denote the corresponding scaled value as 
$\tilde{\tilde{z}_{\|}}$, so $\bar{z}_{\|}=w^{2} \tilde{\tilde{z}_{\|}}$and $\tilde{z_{\|}}$is constant in a scaled spectrum.

In this way we find that the oscillator strength density for any sideband $D f_{l}(w ; \epsilon)(2.22)$ is approximately the static density of $D f_{\text {static }}(w ; \epsilon)$ shifted by a multiple of $a$ along the $w$ axis, and weighted by the appropriate Bessel function:

$$
D f_{\mathrm{rf}}(w ; \epsilon)=\sum_{l=-\infty}^{+\infty} J_{l}^{2}\left(\frac{w \widetilde{F}_{1} \tilde{z}_{\|}}{\tilde{\omega}}\right) D f_{\text {static }}(w-l a ; \epsilon) .
$$

The relevant range of $w$ in the Fourier transform is small enough that we can replace $w$ in the argument of the Bessel function by its value at the midpoint of the scaled scan $w_{0}$ (the range of such a scan is about $10 \%$ of $w_{0}$ ).

Then the sum over $l$ has the structure of a convolution sum. Not surprisingly, the Fourier transform becomes a product,

$$
R_{\mathrm{rf}}(s)=R_{\text {static }}(s) R_{s b}(s) .
$$

The first factor in this product is the recurrence spectrum in static fields only. The second factor in this product,

$$
R_{s b}(s)=\sum_{l=-\infty}^{+\infty} J_{l}^{2}\left(w_{0} \frac{\tilde{F}_{1} \tilde{z}_{\|}}{\tilde{\omega}}\right) e^{-2 \pi i s a l}
$$

may be interpreted as the Fourier transform of a single cluster of levels composed of one progenitor and its sidebands. To see this, suppose the absorption spectrum consisted of a single progenitor that we can place arbitrarily at $w=0$ together with its sidebands at $w=l a$. The oscillator-strength density would then be

$$
D f_{s b}(w)=\sum_{l=-\infty}^{+\infty} J_{l}^{2}\left(w_{0} \frac{\tilde{F}_{1} \tilde{z_{\|}}}{\tilde{\omega}}\right) \delta(w-l a)
$$

and the Fourier transform of this quantity gives the expression (4.17). We can simplify Eq. (4.17) by use of the Besselfunction sum rule [7],

$$
\begin{aligned}
R_{s b}(s) & =\sum_{l=-\infty}^{+\infty} J_{l}^{2}(q) e^{-2 \pi i s a l}=J_{0}[2 q \sin (\pi a s)] \\
& =J_{0}\left(2 q \sin \left(\pi s / s_{s b}\right)\right)=J_{0}\left(2 q \sin \left[\pi\left(T_{\|} / T_{\mathrm{rf}}\right)\left(s / S_{\|}\right)\right]\right) .
\end{aligned}
$$

That gives for the squared absolute value of the Fourier transform

$$
\begin{aligned}
\left|R_{\mathrm{rf}}(s)\right|^{2}= & \left|R_{\text {static }}(s)\right|^{2} J_{0}^{2}\left(\left(2 w_{0} \tilde{F}_{1} \tilde{z}_{\|} / \tilde{\omega}\right)\right. \\
& \left.\times \sin \left[\pi\left(s / S_{\|}\right)\left(T_{\|} / T_{\mathrm{rf}}\right)\right]\right) .
\end{aligned}
$$

The maximum value of the Bessel function is 1 [compare with the intensity conservation rule (2.19)] and the function is slowly varying in $s$, so it generates an "envelope function," smoothly modifying the static recurrence spectrum. Most recurrences are weakened with increasing $F_{1}$, but some are preserved (Fig. 6).

The envelope has a multipeaked structure that is governed by two parameters. The first parameter is $q=w_{0} \widetilde{F}_{1} \tilde{z}_{\|} / \tilde{\omega}$

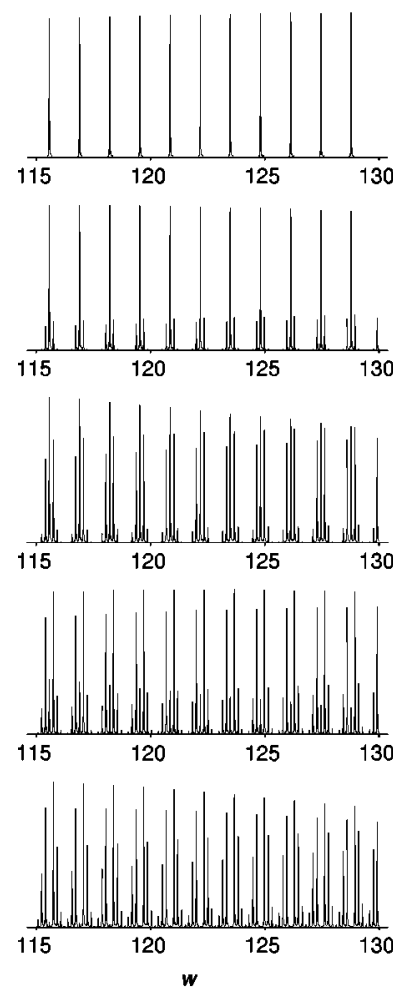

Absorption Spectrum
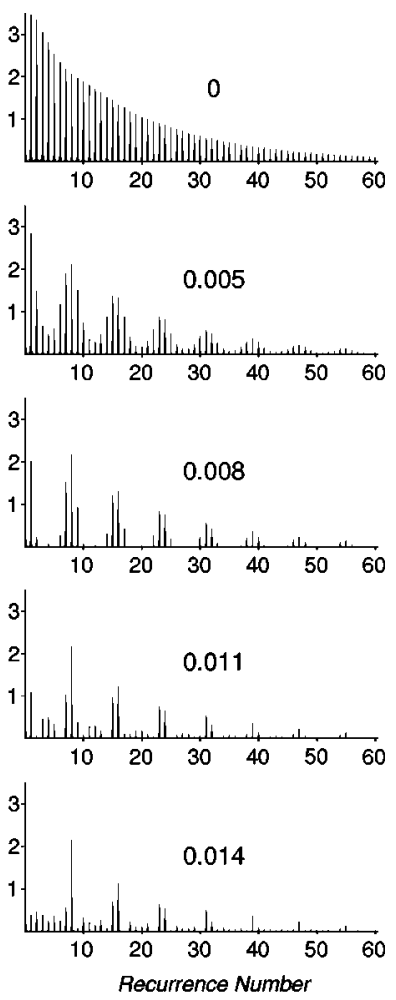

Recurrence Spectrum
FIG. 6. rf field influence on the absorption and recurrence spectra (atomic units are used). At $\epsilon=-0.11$, the static spectrum $\left(\widetilde{F}_{1}\right.$ $=0)$ consists of an almost perfectly periodic sequence of $n_{2}=0$ levels. Its recurrence spectrum is a system of equidistant peaks. When the rf field is turned on, each progenitor (state in the static spectrum) gives rise to a cluster of nearly equally spaced sidebands (the values of $\widetilde{F}_{1}$ are shown above each recurrence spectrum). The sideband spacing is in proportion to $\omega_{\mathrm{rf}}: \omega_{\|}=1: 8$. As $\widetilde{F}_{1}$ increases and the sidebands develop, the static recurrence spectrum is multiplied by an envelope function. This envelope function is the Fourier transform of a single cluster. The envelope preserves recurrences having return time equal to a multiple of the period of the rf field (every eighth peak in our case).

$=F_{1} \bar{z}_{\|} / \hbar \omega$, which appeared earlier [Eqs. (2.16),(2.18)] and which governs the population of sidebands. As $q$ increases, the weights of sidebands increase and the number of significant sidebands increases. The second parameter is $s_{s b}=1 / a$ $=S_{\|} T_{\mathrm{rf}} / T_{\|}$, which is the inverse of the spacing between sidebands and therefore represents the new periodicity that shows up in the Fourier transform at $s_{s b}$ and its multiples. At small $q$, only a few sidebands are significantly populated, so the Fourier transform is a smoothly varying function that is largest at these values of $s$. Such a process is illustrated by Fig. 6. It shows the sharpening of the peaks of the envelope when the amplitude of the rf field (and the number of sidebands) increases.

For any $q$, the Bessel function $J_{0}$ is near unity if the sin contained in its argument is near zero; this happens when $s$ is near any multiple of $s_{s b}$, or

$$
n(s) \equiv s / S_{\|}=m\left(T_{\mathrm{rf}} / T_{\|}\right),
$$

where $m$ is any positive integer. 
In the present case, all recurrences occur with actions $s$ that are close to integer multiples of $S_{\|}$; therefore we can regard $n(s)$ as an integer. This formula then says that the $n$th recurrence survives the disturbance by the rf field if

$$
n T_{\|}=m T_{\mathrm{rf}},
$$

i.e., if its return time is a multiple of the $\mathrm{rf}$ period. (In other words, this recurrence peak coincides with one of the maxima of the envelope.)

One can expect similar effects in an unscaled absorption spectrum in the presence of a weak unscaled rf field because the sidebands of the unscaled spectrum are exactly equally spaced in energy.

\section{Comparison with results of semiclassical theory}

The argument of the Bessel function in Eq. (4.20) is similar but not identical to Eq. (10) of [1], which was derived from closed-orbit theory. The envelope function obtained from that theory was

$$
J_{0}^{2}\left(\widetilde{f} \widetilde{Z}_{1}(\tilde{\omega})\left|\frac{\sin \left(n \tilde{\omega} \widetilde{T}_{1} / 2\right)}{\sin \left(\tilde{\omega} \widetilde{T}_{1} / 2\right)}\right| \widetilde{T}_{1} w\right) .
$$

In this formula $Z_{1}(\omega)$ denotes the time-averaged ac dipole moment of a closed orbit, $Z_{1}=\left(1 / T_{1}\right) \int_{0}^{T_{1}} z(\tau) e^{-i \omega \tau} d \tau$, with period $T_{1}$. Tilde, as usual, means the scaled quantity: $\widetilde{Z}_{1}$ $=Z_{1} w^{-2}, \widetilde{T}_{1}=T_{1} w^{-3}$. In the present notation $\left(\widetilde{f} \rightarrow \widetilde{F}_{1}\right)$. Eq. (4.23) applies to any individual orbit, but in the present case all orbits are close to the parallel orbit, so we can replace $\widetilde{T}_{1}$ by $\widetilde{T}_{\|}$, and $\widetilde{Z}_{1}(\tilde{\omega})$ by $\widetilde{Z}_{\|}(\tilde{\omega})$. Then the envelope function in closed-orbit theory is

$$
J_{0}^{2}\left(\widetilde{F}_{1} \widetilde{Z}_{\|}(\tilde{\omega})\left|\frac{\sin \left(n \tilde{\omega} \widetilde{T}_{\|} / 2\right)}{\sin \left(\tilde{\omega} \widetilde{T}_{\|} / 2\right)}\right| \widetilde{T}_{\|} w\right) .
$$

Equation (4.20) is a low-frequency limit of this formula. If the period of the rf field is long compared to the return time of the orbit, then

$$
\sin \left(\tilde{\omega} \widetilde{T}_{\|} / 2\right) \rightarrow\left(\tilde{\omega} \widetilde{T}_{\|} / 2\right)=\left(\pi \widetilde{T}_{\|} / \widetilde{T}_{\mathrm{rf}}\right)
$$

and the ac dipole moment of the orbit goes to the static dipole moment,

$$
\widetilde{Z}_{\|}(\tilde{\omega}) \rightarrow \widetilde{Z}_{\|}(0) \equiv \tilde{\tilde{z}_{\|}}
$$

If also the measured range of $w$ is small, we can replace $w \rightarrow w_{0}$ to obtain

$$
J_{0}^{2}\left(\left(2 \widetilde{F}_{1} \tilde{z_{\|}} / \tilde{\omega}\right) \sin \left(\pi n T_{\|} / T_{\mathrm{rf}}\right) w_{0}\right)
$$

In this formula $n$ is an integer, referring to the $n$th repetition of the parallel orbit. In the present case all the recurrences occur near reduced actions $s$, which are multiples of $S_{\|}, s$ $=n S_{\|}$, or $n=s / S_{\|}$at a recurrence. Thus we arrive at Eq. (4.20) as a low-frequency approximation to Eq. (4.24). [Of the two, we believe that Eq. (4.24), from closed-orbit theory, is the more accurate and the more general formula [12].]
We have therefore arrived at comparable formulas in two different ways. These two derivations emphasize different aspects of the physics of the problem. (a) In the semiclassical framework, the parameter

$$
e F_{1}|Z(\omega)| T_{\|} / \hbar
$$

is a measure of the change of action on the parallel orbit that is induced by the rf field. In the semiquantal framework, the parameter

$$
q=e F_{1} \bar{z} T_{\|} / \hbar
$$

appears in Eq. (4.16) as the argument of the Bessel function $J_{l}^{2}(q)$. Therefore $q$ is connected to the relative strength of sidebands; when $q \sim l$, the $l$ th sideband makes the largest contribution.

(b) If in Eq. (4.20) we consider $n(s)$ to be an integer, then the same sin function appears in both semiclassical and semiquantal formulas,

$$
\sin \left(\pi n T_{\|} / T_{\mathrm{rf}}\right)
$$

and it vanishes for those orbits having return time equal to a multiple of the rf period. In the semiclassical theory, those orbits have their action unchanged by the rf field. In the semiquantal theory, if we examine Eq. (4.19), the exponential factor is

$$
e^{2 \pi i s a l}=e^{2 \pi i \ln (s) T_{\|} / T_{\mathrm{rf}}} .
$$

At values of $s$ such that $n(s) T_{\|} / T_{\text {rf }}$ is an integer, then the sidebands all add in phase, and Eq. (2.19) tells us that the recurrence survives the perturbation. In this way the spacing between progenitors becomes unimportant (as the progenitors disappear), while the nearly constant spacing between sidebands dominates the Fourier transform.

Thus the semiclassical closed-orbit theory and the present semiquantal theory give complementary interpretations of the observed phenomena, one in terms of the change of action on closed orbits, and the other in terms of the development of clusters of quasienergy levels.

\section{CONCLUSION}

Following is a summary of the theory developed here. (1) Energy levels in a static electric field can be calculated from familiar Bohr-Sommerfeld (WKB) quantization conditions; each level is labeled by quantum numbers $\left(n_{1}, n_{2}, m\right)=(\mathbf{n}, m)$. (2) Intensities (oscillator strengths) in the static field can be calculated from a new semiclassical formula that we derived recently [6]. (3) With the rf field added, each Stark energy level, which we call a progenitor, is split into a series of equally spaced sidebands having energy $E_{\mathbf{n}, l}=E_{\mathbf{n}}+l \hbar \omega$. These are the quasienergy states. (4) The intensity in each sideband is that for the unperturbed progenitor times a Bessel function $J_{l}$ of an argument that depends on n. (5) These quasienergies and their intensities can be calculated in scaled variables.

The above calculation of the spectrum of quasienergy levels and their intensities (in scaled or unscaled variables) is what we call the semiquantal method. Calculations show that the resulting absorption spectrum and its Fourier transform 
are in good agreement with experiments, and with semiclassical calculations reported in [1].

This method, however, does not give much physical insight, and, in particular, we cannot see directly why certain recurrences are weakened while others are preserved by the rf field. Additional approximations are needed to get a physical understanding of the results. With these additional approximations, we show the following: (6) The scaledvariables absorption spectrum is nearly periodic in $w$. Static energy levels are almost uniformly spaced in $n_{1}$, and sidebands are almost uniformly spaced in $l$. (7) The absorption spectrum in the rf field is a superposition of the spectrum of progenitors and the spectrum of sidebands; this sum has the form of a convolution. Therefore the recurrence spectrum in the rf field is a product,

$$
R_{\mathrm{rf}}(s)=R_{\text {static }}(s) R_{s b}(s) .
$$

The first factor is the recurrence spectrum in the static field. The second factor gives a more-slowly-varying envelope function, which is the Fourier transform of the spectrum of sidebands arising from a single progenitor.

(8) This envelope function $R_{s b}(s)$ turns out to be a Bessel function $J_{0}$ of a complicated argument. The argument is the low-frequency limit of that obtained in the semiclassical theory. That envelope function contains two parameters: one is related to the strength of the sidebands relative to the progenitor (and to the number of significant sidebands), and the other is related to the spacing of the sidebands. The spacing parameter $s_{s b}$ has the effect of selecting recurrences that survive the perturbation. As the strength parameter $q$ increases, the preservation of recurrences becomes more selective, conserving recurrences in an ever narrower band.

(9) The evenly spaced sequence of sidebands produces in the scaled absorption spectrum a new periodicity that is not present in the static spectrum. This periodicity of course shows up in the Fourier transform. Its effect on the recurrence spectrum is to preserve those recurrences whose return time is a multiple of the rf period.

Thus we give a semiquantal explanation of the most striking feature of the experimental observations.

\section{ACKNOWLEDGMENTS}

This work was supported by the National Science Foundation and the U.S. Office of Naval Research. All experimental data have been generously provided to us by $\mathrm{N}$. Spellmeyer and D. Kleppner, and we thank them for many helpful discussions, as well as M. Haggerty and J. Gao.

\section{APPENDIX}

In this Appendix we list expressions for some useful semiclassical functions via complete elliptic integrals of the first kind $K(m)$ and the second kind $E(m)$,

$$
\begin{aligned}
& K(m)=\int_{0}^{\pi / 2}\left(1-m \sin ^{2} t\right)^{-1 / 2} d t \\
& E(m)=\int_{0}^{\pi / 2}\left(1-m \sin ^{2} t\right)^{1 / 2} d t
\end{aligned}
$$

There exists a very efficient method of the arithmeticalgeometrical mean to calculate these integrals [7].

\section{Action variables}

The action variables for bounded motion in the Stark problem have the form [cf. Eqs. (2.4),(2.5); all notation is defined in Sec. II]

$$
\begin{aligned}
I_{u} & =w S_{u}=\frac{1}{2 \pi} \int_{o s c} p_{u} d u \\
& =\frac{1}{\pi} \int_{0}^{u_{0}} \sqrt{2(1+\beta)+2 E u^{2}-F u^{4}} d u, \\
I_{v} & =w S_{v}=\frac{1}{2 \pi} \int_{o s c} p_{v} d v \\
& =\frac{1}{\pi} \int_{0}^{v_{0}} \sqrt{2(1-\beta)+2 E v^{2}+F v^{4}} d v .
\end{aligned}
$$

These classical formulas are easily derived from the quantum Schrödinger equation using the Langer-modified WKB approximation.

A simple transformation allows us to express the action variables via elliptic integrals defined above:

$$
\begin{aligned}
S_{u}= & \frac{2^{1 / 2}}{3 \pi}\left[\epsilon^{2}+2(1+\beta)\right]^{1 / 4}\left[\left(\sqrt{\epsilon^{2}+2(1+\beta)}-\epsilon\right) K\left(m_{u}\right)\right. \\
& \left.+2 \epsilon E\left(m_{u}\right)\right], \\
S_{v}= & -\frac{2}{3 \pi} \sqrt{\sqrt{\epsilon^{2}-2(1-\beta)}-\epsilon}\left[\sqrt{\epsilon^{2}-2(1-\beta)} K\left(m_{v}\right)\right. \\
& \left.+\epsilon E\left(m_{v}\right)\right],
\end{aligned}
$$

where

$$
\begin{gathered}
m_{u}=\frac{1}{2}\left(1+\frac{\epsilon}{\sqrt{\epsilon^{2}+2(1+\beta)}}\right), \\
m_{v}=-\frac{\sqrt{\epsilon^{2}-2(1-\beta)}+\epsilon}{\sqrt{\epsilon^{2}-2(1-\beta)}-\epsilon} .
\end{gathered}
$$

\section{Derivatives of action variables}

Some key formulas of this article [see, for example, Eqs. (2.15) and (2.22)] contain Jacobians of action variables $I_{u}, I_{v}$ over the integrals of motion $E, \beta$ and external electric field strength $F$. We list here expressions for these derivatives via elliptic integrals $K(m)$ and $E(m)$. These expressions make possible an efficient calculation of Jacobians.

The separation of motion in parabolic coordinates (2.3) involves the introduction of a new time variable $\tau$ that is related with the real laboratory time $t$ by the relation $d \tau$ $=d t / r$. In parabolic momenta $p_{u}$ and $p_{v}$ the combinations $2(1+\beta)$ and $2(1-\beta)$, respectively, play the role of energy. That is why the derivatives of the action variables (A3) and (A4) with respect to the separation constant $\beta$ give, to a constant factor, the "canonical periods" of motion (in $\tau$ ) 
along the parabolic coordinates:

$$
\begin{gathered}
\partial I_{u} / \partial \beta=\tau_{u} / 4 \pi, \\
\partial I_{v} / \partial \beta=-\tau_{v} / 4 \pi
\end{gathered}
$$

with

$$
\begin{gathered}
\frac{\partial I_{u}}{\partial \beta}=\frac{\tau_{u}}{4 \pi}=\frac{w}{4 \pi} \frac{2^{3 / 2} K\left(m_{u}\right)}{\left[\epsilon^{2}+2(1+\beta)\right]^{1 / 4}}, \\
\frac{\partial I_{v}}{\partial \beta}=-\frac{\tau_{v}}{4 \pi}=-\frac{w}{4 \pi} \frac{4 K\left(m_{v}\right)}{\sqrt{\sqrt{\epsilon^{2}-2(1-\beta)}-\epsilon}},
\end{gathered}
$$

where the parameters $m_{u}$ and $m_{v}$ are defined by (A7) and (A8).

These quantities do not represent real time, but the quasitime that is used for regularizing the motion in parabolic coordinates.

The derivatives of these action variables with respect to energy are also useful. These derivatives give quantities that have units of real, laboratory time, and we denote them $R_{u}$ and $R_{v}$ :

$$
\begin{gathered}
\partial I_{u} / \partial E=R_{u} / 2 \pi, \\
\partial I_{v} / \partial E=R_{v} / 2 \pi
\end{gathered}
$$

with

$$
\begin{aligned}
R_{u}= & w^{3} \frac{\sqrt{2}}{\left[\epsilon^{2}+2(1+\beta)\right]^{1 / 4}}\left[2 \sqrt{\epsilon^{2}+2(1+\beta)} E\left(m_{u}\right)\right. \\
& \left.-\left(\sqrt{\epsilon^{2}+2(1+\beta)}-\epsilon\right) K\left(m_{v}\right)\right], \\
R_{v}= & w^{3} 2 \sqrt{\sqrt{\epsilon^{2}-2(1-\beta)}-\epsilon}\left[K\left(m_{v}\right)-E\left(m_{v}\right)\right] .
\end{aligned}
$$

However, these formulas must be interpreted with caution. In general $R_{u}$ and $R_{v}$ do not represent the natural periods of motion. The period associated with $u$ motion is $2 \pi /\left(\partial E / \partial I_{u}\right)_{I_{v}}$ whereas $R_{u}$ is $2 \pi /\left(\partial E / \partial I_{u}\right)_{\beta}$. These are not equal.

It follows that there are different rules of operation with canonical periods $\tau_{u, v}$ and "ersatz-real-time-periods" $R_{u, v}$. If we deal with a closed orbit with $m$ oscillations along the $u$ coordinate and $n$ along the $v$ coordinate before closure, we can write that $m \tau_{u}=n \tau_{v}$ and the total canonical time $\tau$ between the closures is equal either $m \tau_{u}$ or $n \tau_{v}$. As for the real laboratory time $t=R$ between closures, it is equal to $R$ $=m R_{u}+n R_{v}$ where, generally, $m R_{u} \neq n R_{v}$. When the ejection angle $\theta \rightarrow 0$, the ratio of canonical periods $\tau_{v} / \tau_{u}$ has a nonzero limit, but the ratio $R_{v} / R_{u}$ tends to zero. Therefore in that limit, $R_{u}$ is the real return time of the parallel orbit, which we call $T_{\|}$.

The derivatives with respect to external electric field strength can be calculated according to the following formulas:

$$
\begin{aligned}
\frac{\partial I_{u}}{\partial F}= & w^{5} \frac{\left[\epsilon^{2}+2(1+\beta)\right]^{1 / 4}}{2^{3 / 2} 3 \pi}\left[\left(4 \epsilon-\sqrt{\epsilon^{2}+2(1+\beta)}\right.\right. \\
& \left.\left.-\frac{3 \epsilon^{2}}{\sqrt{\epsilon^{2}+2(1+\beta)}}\right) K\left(m_{u}\right)-8 \epsilon E\left(m_{u}\right)\right], \quad
\end{aligned}
$$

$$
\begin{aligned}
\frac{\partial I_{v}}{\partial F}= & w^{5} \frac{\sqrt{\sqrt{\epsilon^{2}-2(1-\beta)}-\epsilon}}{6 \pi}\left[\left(\sqrt{\epsilon^{2}-2(1-\beta)}-3 \epsilon\right) K\left(m_{v}\right)\right. \\
& \left.+4 \epsilon E\left(m_{v}\right)\right] .
\end{aligned}
$$

\section{Parameters of the parallel orbit}

The parallel orbit corresponds to zero ejection angle $(\theta$ $=0, \beta=1$ ). Of course, this orbit by itself does not represent any quantum state because that would violate the uncertainty principle. However, the parameters of this orbit are relevant to our calculations because they serve as good approximation to parameters of the states that are stretched uphill along the field axis (the states with $n_{2}=0$ ). So we have

$$
\omega_{\|}=2 \pi / R_{\|}(\beta=1) \equiv 2 \pi / T_{\|} .
$$

This expression has a very simple limit when $\epsilon \rightarrow 0$ :

$$
\omega_{\|}=3.71 / w^{3} \text {. }
$$

As for $\bar{z}_{\|}$, its expression (2.15) also becomes more simple when $\beta=1$ :

$$
\bar{z}_{\|}=-\left(\partial I_{u} / \partial F\right) / R_{u}(\beta=1),
$$

where the expressions (A17) and (A15) with $\beta=1$ should be used. These expressions show that the scaled quantity $\tilde{z}_{\|}$ $=\bar{z}_{\|} w^{-2}$ depends only on the scaled energy $\epsilon$ and therefore is constant along the scaled scan. Again, in the limit $\epsilon \rightarrow 0$ we have a very simple result,

$$
\tilde{z_{\|}}=0.116 \text {. }
$$

[1] N. Spellmeyer, D. Kleppner, M. R. Haggerty, V. Kondratovich, J. B. Delos, and J. Gao, Phys. Rev. Lett. 79, 1650 (1997).

[2] Y. Zhang, M. Ciocca, L.-W. He, C. E. Burkhardt, and J. J. Leventhal, Phys. Rev. A 50, 1101 (1994).

[3] Y. Zhang, M. Ciocca, L.-W. He, C. E. Burkhardt, and J. J. Leventhal, Phys. Rev. A 50, 4608 (1994).

[4] M. L. Du and J. B. Delos, Phys. Rev. A 38, 1896 (1988); 38, 1913 (1988).
[5] The reader can find a review of WKB methods as they apply to the quantization in strong electric field by C. W. Clark, K. T. Lu, and A. F. Starace, in Progress in Atomic Spectroscopy, edited by H. J. Beyer and H. Kleinpoppen (Plenum Press, New York, 1984), Part C, Chap. 7.

[6] V. Kondratovich and J. B. Delos, Phys. Rev. A 56, R5 (1997).

[7] F. Olver, in Handbook of Mathematical Functions, Natl. Bur. Stand. Appl. Math. Ser. No. 55, edited by M. Abramowitz and 
I. Stegun (U.S. GPO, Washington, D.C., 1964).

[8] N. Spellmeyer and D. Kleppner (private communication).

[9] N. Spellmeyer (personal communication) tells us that such a shift is credible. There is an uncertainty of about $10 \mathrm{mV} / \mathrm{cm}$ in the electric field in experiment, and a stray electric field with a similar size. At $w=150$, a $20-\mathrm{mV} / \mathrm{cm}$ uncertainty gives an uncertainty in position of a resonance on the $w$ axis of 0.08 .

[10] L. D. Landau and E. M. Lifshitz, Quantum Mechanics: Nonrelativistic Theory, 3rd ed. (Pergamon Press, Oxford, New York, 1977).

[11] For the experimental conditions in [1], we have $n_{1} \sim 110, n_{2}$ $\leqslant 7$; trajectories with larger $n_{2}$ go over the barrier. Therefore we find that the scaled spectrum is nearly periodic. (In the case we consider, the spacing of levels in $n_{1}$ is uniform to about one part in $10^{6}$, while the spacing in $n_{2}$ is uniform to a few percent.)

[12] As a general rule, a semiquantal treatment like the one used here should be more accurate than a semiclassical treatment such as closed-orbit theory. However, in the present case, we have made a number of additional approximations to arrive at Eq. (4.20), the most important of which is an adiabatic approximation. The closed-orbit formulation that led to Eq. (4.24) did not use an adiabatic approximation, so we believe Eq. (4.24) to be, in principle, more general and more accurate than Eq. (4.20). 